\section{PWE-038 ENDOSCOPIC RESECTION OF AMPULLARY AND DUODENAL ADENOMAS: A TERTIARY REFERRAL CENTRE EXPERIENCE}

${ }^{1} \mathrm{E}$ Hossain*, ${ }^{2} \mathrm{M}$ Abdelrahim, ${ }^{2} \mathrm{~L}$ Varytimiadis, ${ }^{2} \mathrm{~A}$ Alkandari, ${ }^{2} \mathrm{P}$ Goggin, ${ }^{2} \mathrm{~S}$ Arndtz,

${ }^{2} \mathrm{P}$ Bhandari, ${ }^{2} \mathrm{~S}$ Subramaniam. 'Queen Alexandra Hospital, Portsmouth; ' ${ }^{2} \mathrm{QA}$ Hospital, Portsmouth

\subsection{6/gutjnl-2019-BSGAbstracts.325}

Introduction Duodenal and ampullary adenomas have the potential for malignant transformation to carcinomas by an adenoma-to-carcinoma sequence as seen elsewhere in the gastrointestinal tract. Endoscopic resection of these lesions is increasingly being used in place of invasive surgery. However, endoscopic resection in the duodenum can be challenging due to its anatomy and may be associated with a higher risk of complications. This study aims to evaluate the safety and efficacy of endoscopic resection of such lesions.

Methods All patients with ampullary and large non-ampullary (>10 mm) duodenal polyps who underwent endoscopic resection between February 2008 and January 2019 in a single tertiary referral centre were included in the study. A retrospective analysis on data including demographics, size, histology, location, method of resection and complications was performed.

Results There were a total of 112 patients referred for endoscopic therapy. Of these 112 patients, 37 underwent ampullectomy and 85 patients were referred for resection of nonampullary duodenal polyps $>10 \mathrm{~mm}$.

In patients who underwent ampullectomy, 27 (73\%) had a submucosal injection, whereas pancreato-duodenal stent was placed in $26(70 \%)$ of patients. 7 (20\%) patients had adjunct tissue ablation (APC). $62 \%$ of the lesions were pure ampullary, whereas 38\% polyps involved the duodenum.

In the 85 patients who were referred with a non ampullary duodenal lesion, 58 (68\%) underwent endoscopic mucosal resection (EMR) and 27 (32\%) had knife assisted resection (KAR). Majority of the lesions (70) were found in D2 (82\%).

Further characteristics have been summarized in table 1

\begin{tabular}{lll}
\multicolumn{2}{l}{ Abstract PWE-038 Table 1 } & \\
\hline & Non ampullary duodenal resections & Ampullectomies \\
\hline Number & 85 & 37 \\
Median age & 64 years [IQR 27-87] & 66 years [IQR 50-83] \\
Mean size & $25.3 \mathrm{~mm}$ & $21.6 \mathrm{~mm}$ \\
Diagnosis of FAP & $9.4 \%$ & $13.5 \%$ \\
En bloc resection & $40 \%$ & $43.2 \%$ \\
Median follow up & $38 \mathrm{months}$ & $16 \mathrm{months}$ \\
Recurrence & $7 \%$ & $24.3 \%$ \\
Bleeding & $8 \%$ & $5.4 \%$ \\
Perforation & $2 \%$ & $0 \%$ \\
Lesions $>30 \mathrm{~mm}$ & $38 \%$ & $32 \%$ \\
\hline
\end{tabular}

All complications were managed endoscopically The majority of the recurrences were treated successfully with subsequent endoscopic resection. No procedure related mortality was noted in this cohort.

Conclusions Endoscopic resection of duodenal and ampullary lesions is a safe and effective technique for complete removal of these polyps. The risk of complications is similar in ampullary and non-ampullary resections, however, the risk of recurrence is higher in patients with ampullary adenomas. Nevertheless, we demonstrated that all complications and most recurrences were successfully managed endoscopically.

\section{Nutrition}

Orals

\section{OTU-27 THE HIDDEN BURDEN OF EMERGENCY DEPARTMENT ATTENDANCES FOR LATE ENTERAL TUBE FEEDING COMPLICATIONS}

Mrs Diane Barrett*, Sue Merrick, Vincent Li, JingJing Ruan, Aravinth Murugananthan Helen Steed. The Royal Wolverhampton NHS Trust, Wolverhampton, UK

\subsection{6/gutjnl-2019-BSGAbstracts.326}

Introduction Late complications related to enteral tube feeding are little documented in the literature. One 10 year study reports a longitudinal rate of displacement of up to $12 \%{ }^{1}$. Here we investigate the hidden burden of community enteral tube feeding on the Emergency Department (ED).

Method We undertook a retrospective review of attendances to the emergency department of the district general hospital in Wolverhampton with gastrostomy and jejunostomy tube complications. ED discharge coding was reviewed over an 18 month period to identify patients attending ED with a gastrostomy or jejunostomy tube. Electronic medical records were then reviewed to determine if the primary reason for attendance was related to the enteral feeding tube.

Results In an 18 month period 139 attendances were recorded, $56 \%$ of cases were male patients. Fifty patients came from nursing homes, 8 from residential homes, 2 from another hospital and 79 (57\%) from their own homes.

One hundred and nine were completely dependent on the enteral tube for all nutrition and hydration, 8 required it for fluids only, 2 for nutrition only and 20 were not completely dependent on the enteral tube.

The type of tube prompting attendance is shown in table 1

\begin{tabular}{ll} 
Abstract 0 TU-27 Table 1 & \\
\hline Type of enteral tube & No \\
\hline PEG & 46 \\
RIG & 26 \\
PEG-J & 8 \\
Transgastric BGT & 7 \\
Jejunostomy & 28 \\
Low profile & 8 \\
BGT & 16 \\
\hline
\end{tabular}

$(\mathrm{PEG}=$ percutaneous endoscopic gastrostomy, $\mathrm{RIG}=$ radiologically inserted gastrostomy, $\mathrm{J}=$ jejunostomy, $\mathrm{BGT}=$ balloon gastrostomy tube)

The problems causing the ED attendance are shown in table 2 .

Where in hours was defined as 9-5 Monday to Friday and all other times were defined as out of hours 57 attending during standard working hours of the nutrition support team and 82 attended when the support team were not available, out of hours. 


\begin{tabular}{lll}
\multicolumn{2}{l}{ Abstract OTU-27 Table } & $\mathbf{2}$ \\
\hline Problem & No & Percentage \\
\hline Dislodged & 59 & $42 \%$ \\
Blocked & 22 & $16 \%$ \\
Infected & 12 & $9 \%$ \\
Leakage & 13 & $10 \%$ \\
Split tube & 7 & $5 \%$ \\
Pain & 4 & $3 \%$ \\
Bleed & 4 & $3 \%$ \\
Damaged tube & 7 & $5 \%$ \\
Other & 11 & $7 \%$ \\
\hline
\end{tabular}

Conclusion Using current tariffs the cost of the attendances to ED are $£ 29,000$ over the analysed period, with a significant proportion of patients coming from their own home and 60\% out of hours. More work on education and supporting carers to resolve problems themselves could reduce the burden on busy EDs.

\section{OWE-15 ADVANCED LIVER FUNCTION ASSESSMENT IN PATIENTS WITH INTESTINAL FAILURE ON LONG-TERM PARENTERAL NUTRITION}

${ }^{1}$ Elisabeth Blüthner*, ${ }^{2}$ Jan Bednarsch, ${ }^{1,3}$ Ulrich-Frank Pape, ${ }^{1}$ Mirjam Karber, 1,3 Sebastian Maasberg, ${ }^{4}$ Undine Gerlach, ${ }^{4,5}$ Andreas Pascher, ${ }^{1}$ Bertram Wiedenmann, ${ }^{4} J$ ohann Pratschke, ${ }^{4,6}$ Martin Stockmann. ${ }^{1}$ Department of Gastroenterology and Hepatology, Charité University Medicine, Berlin, Germany; ${ }^{2}$ Department of General, Visceral and Transplantation Surgery, University Hospital Aachen, Aachen, Germany; ${ }^{3}$ Department of Internal Medicine and Gastroenterology, Asklepios Klinik St. Georg, Hamburg, Germany; ${ }^{4}$ Department of Surgery, Charité University Medicine, Berlin, Germany; ${ }^{5}$ Department of General, Visceral and Transplantation Surgery, Münster University Hospital, Münster, Germany; ${ }^{6}$ Department of General, Visceral and Vascular Surgery, Evangelisches Krankenhaus Paul Gerhardt Stift, Lutherstadt Wittenberg, Germany

10.1136/gutjnl-2019-BSGAbstracts.327
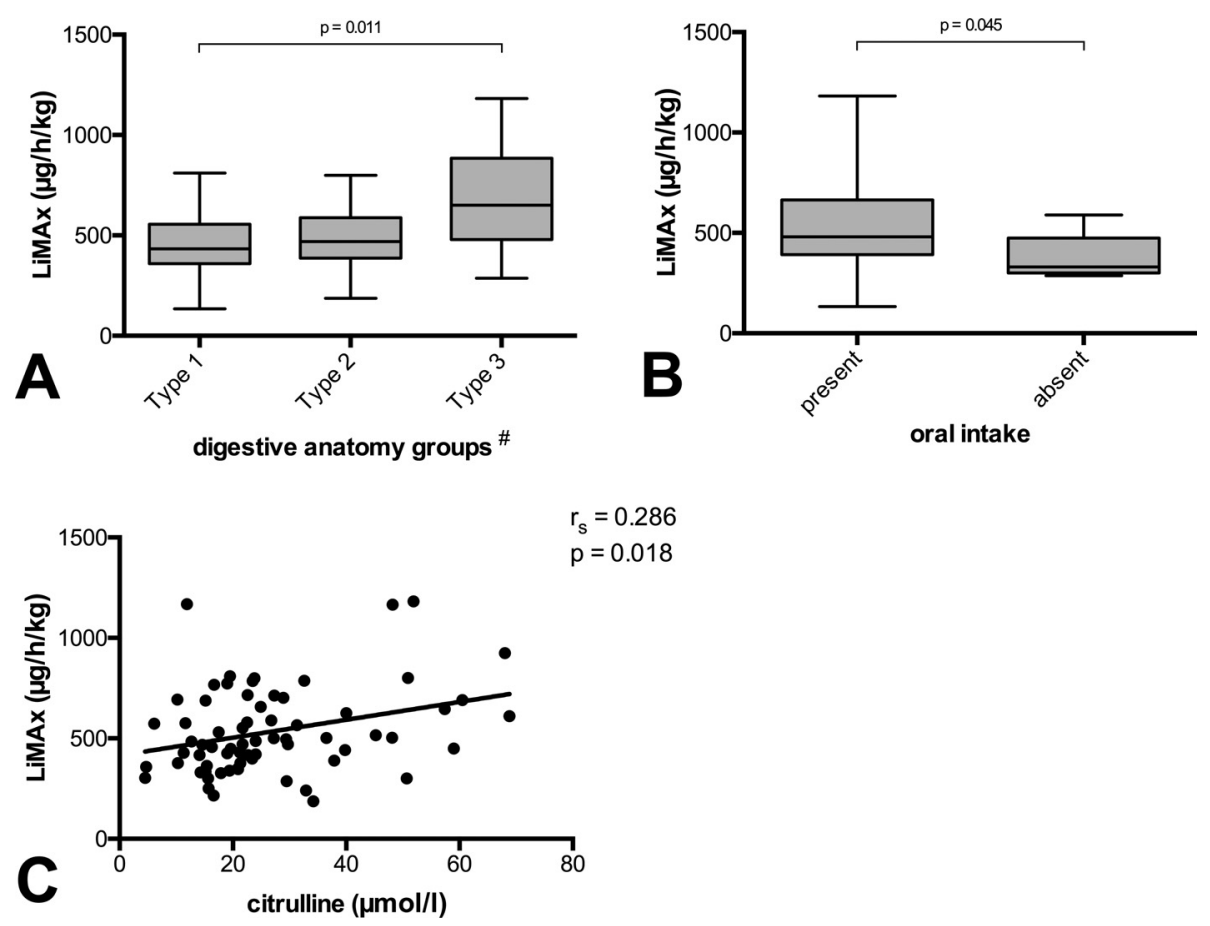

Abstract OWE-15 Figure 1 Parameters independently associated with LiMAx test A: digestive anatomy groups; B: oral intake; C: citrulline level
Introduction Intestinal failure associated liver disease (IFALD) is one of the leading complications and causes of deaths in adult patients receiving home parenteral nutrition for chronic intestinal failure (CIF). Early diagnosis of IFALD is key to alleviate the progression of hepatic dysfunction. The aim of this study was to evaluate the capability of noninvasive liver function tests.

Methods 90 adult patients with CIF receiving long-term home parenteral nutrition were included in a prospective cross-sectional study at our department between 2014 and 2017. All participants underwent dynamic liver function assessment (maximum liver function capacity [LiMAx] test, indocyanine green [ICG] test), transient elastography (FibroScan), blood tests and comprehensive nutritional status assessment. Univariate and multivariable analysis were performed to identify predictors of liver function.

Results LiMAx, ICG test, and FibroScan highly correlated with standard liver function tests. Multivariable analysis identified intact ileum $(\mathrm{B}=520.895 ; \mathrm{p}=0.010)$, digestive anatomy type $3(\mathrm{~B}=75.612 ; \mathrm{p}=0.025)$, citrulline level $(\mathrm{B}=3.428$; $\mathrm{p}=0.040)$, parenteral olive oil intake $(\mathrm{B}=-0.570 ; \mathrm{p}=$ $0.043)$, and oral intake $(B=182.227 ; \mathrm{p}=0.040)$ as independent risk factors affecting liver function determined by LiMAx test. ICG test and FibroScan showed no correlation with gastrointestinal and nutrition-related parameters.

Conclusions We demonstrate that LiMAx test is significantly associated with widely accepted risk factors for IFALD by multivariable analysis, whereas ICG test and FibroScan failed to show significant correlations. Liver function assessment by LiMAx test may have the potential to diagnose IFALD at an early stage and improve long-term outcome in patients with long-term parenteral nutrition. 